\title{
Biofortificación con cobre en el forraje verde de trigo
}

\section{Biofortification with copper in green wheat forage}

\section{Anadelia Antonio-Medina ${ }^{1}$, \\ Leticia Romana Gaytán- \\ Alemán ${ }^{1}[\mathbb{0}$ \\ Urbano Nava-Camberos 2 , \\ Sergio Iban Mendoza- \\ Pedroza $\sqrt[3]{ }$, \\ Francisco Gerardo Veliz- \\ Deras" 10 \\ Gerardo Arellano-Rodríguez 1 [] \\ ${ }^{1}$ Postgrado Ciencias \\ en \\ Producción Agropecuaria. Univer- \\ sidad Autónoma Agraria Antonio \\ Narro UL, Periférico Raúl López \\ Sánchez S/N, Valle Verde, CP. \\ 27054. Torreón, Coahuila, México. \\ ${ }^{2}$ Facultad de Agricultura y \\ Zootecnia/Facultad de Ciencias \\ Biológicas, Universidad Juárez del \\ Estado de Durango, CP. 35000. \\ Gómez Palacio, Durango, México. \\ ${ }^{3}$ Programa de Postgrado \\ en Recursos Genéticos \\ Productividad-Ganadera. Cole- \\ gio de Posgraduados, Campus \\ Montecillo. CP. 56230. Montecillo, \\ Estado de México, México.}

*Autor de correspondencia: letygaytan78@gmail.com

\section{Artículo científico}

Recibido: 24 de febrero 2021

Aceptado: 26 de octubre 2021

Como citar: Antonio-Medina

A, Gaytán-Alemán LR, Nava-

Camberos U, Mendoza-Pedroza

SI, Veliz-Deras FG, Arellano-

Rodríguez G (2021) Biofortifi-

cación con cobre en el forraje

verde de trigo. Ecosistemas

y Recursos Agropecuarios

Núm. Esp. II: e2885. DOI:

10.19136/era.a8nll.2885
RESUMEN. Por la importancia que tienen los minerales en la nutrición se evaluó la acumulación de cobre $(\mathrm{Cu})$ en el forraje verde hidropónico de trigo $(\mathrm{FVH})$ en diferentes soluciones y modificaciones en el pH. Se utilizó un arreglo factorial completamente al azar de $3 \times 4 \times 3$ con tres repeticiones. Cada charola representó una unidad experimental, los factores de estudio fueron tres diferentes soluciones, cuatro tratamientos de cobre y tres niveles de $\mathrm{pH}$; factor $\mathrm{A}$, agua de la llave (ALI), solución nutritiva (SN) (método de Steiner) con agua destilada (AD) y solución nutritiva (SN) con agua de llave (ALI); factor B, productos de $\mathrm{Cu}(4,1.3,0.28$ y $25 \%)$ $y$ factor $\mathrm{C} \mathrm{pH}$ (5.0, 6.0 y 7.8). Las variables evaluadas fueron: contenido de cobre $y$ análisis bromatológicos. Los resultados fueron sometidos a un análisis de varianza y se realizaron las separaciones de medias mediante la prueba de Tukey $(\alpha \pm$ 0.05. Existió una mayor absorción de cobre en la interacción de Agua de llave (ALI) con respecto al fertilizante $(\mathrm{F})$ en concentración de 1.30\% Cu-EDTA presentando una concentración de $5.51 \mathrm{ppm}$, y en las soluciones de agua destilada (AD) con solución nutritiva (SN) y pH 6 mostrando una absorción de 3.94 ppm. El contenido de cobre en el forraje modifico FDA y FDN, estos resultados pueden ser de utilidad en estudios futuros para realizar más ensayos sobre los caracteres bioquímicos relacionados con la digestibilidad del forraje, y en la biofortificación.

Palabras clave: Agua de llave, minerales, $\mathrm{pH}$, semillas, solución nutritiva.

ABSTRACT. Due to the importance of minerals in nutrition, it was decided to evaluate the accumulation of copper $(\mathrm{Cu})$ in the hydroponic green forage of wheat $(\mathrm{FVH})$ in different solutions and modifications in the $\mathrm{pH}$. A completely random factorial arrangement of $3 \times 4 \times 3$ with three repetitions was used. Each tray represented an experimental unit, the study factors were three different solutions, four copper treatments and three $\mathrm{pH}$ levels; factor $\mathrm{A}$, tap water (ALI), nutrient solution (SN) (Steiner's method) with distilled water (AD) and nutrient solution (SN) with tap water (ALI); factor $\mathrm{B}$, products of $\mathrm{Cu}(4,1.3,0.2$ and $25 \%)$ and factor $\mathrm{C} \mathrm{pH}$ (5.0, 6.0 and 7.8). The variables evaluated were: copper content and bromatological analysis. The results were subjected to an analysis of variance and the mean separations were carried out using the Tukey test $(\alpha \pm 0.05)$. There was a greater absorption of copper in the interaction of tap water (ALI) with respect to fertilizer (F) in concentration of $1.30 \%$ Cu-EDTA presenting a concentration of $5.51 \mathrm{ppm}$, and in the solutions of distilled water (AD) with nutritive solution (SN) and $\mathrm{pH} 6$ showing an absorption of $3.94 \mathrm{ppm}$. The copper content in the forage modified FDA and FDN, these results may be useful in future studies to carry out more tests on the biochemical characteristics related to forage digestibility, and in biofortification.

Key words: Tap water, minerals, $\mathrm{pH}$, seeds, nutrient solution. 


\section{INTRODUCCIÓN}

La Comarca Lagunera presenta suelos ligeramente alcalinos, con pH mayores de 7.5 (García et al. 2017). Los suelos con pH por encima de 7 o 7.5 presentan carencia de micronutrientes en las plantas (Medina et al. 2000), debido a que su alto contenido de carbonato cálcico impide que la planta pueda absorber gran parte de los nutrientes (Yescas et al. 2018). Las deficiencias de micronutrientes en los suelos se ha generalizado en el mundo, lo que es un factor limitante para la obtención de altos rendimientos en los cultivos (Masunaga y Marques 2018). La necesidad de cultivar ciertas plantas en áreas desérticas puede ser un desafío debido al clima extremo y suelos pobres, pocas especies de plantas prosperan en tales situaciones y a menudo no se usan como fuente de alimento (Palande et al. 2018).

La hidroponía es una alternativa para el uso eficiente del agua y los fertilizantes en los cultivos (Moreno et al. 2015). También existen otras técnicas, como la biofortificación (Garg et al. 2018) que interviene en el incremento, y toleración de micronutrientes (metales pesados) en los cultivos (DuarteZaragoza et al. 2020, García-González et al. 2020), como vitaminas y minerales, que se transportan dentro de la planta y bioacumulan en los tejidos comestibles (Acosta-Estrada et al. 2019). Lo que se ha documentado en algunos cultivos como el trigo (Chugh y Dhaliwal 2013). En la biofortificación existen tres enfoques: agronómico, convencional y transgénico; en el agronómico se emplea la estrategia de fertilización para proporcionar aumentos temporales de micronutrientes a través de los fertilizantes (Saltzman et al. 2016), mientras que el mejoramiento de los cultivos y la biotecnología representan las estrategias para el convencional y transgénico, respectivamente (Garg et al. 2018). La combinación de las dos técnicas, la hidroponía y la biofortificación agronómica, podría ser una alternativa viable para la producción agrícola y en el futuro poder manejar la producción de alimentos (Garg et al. 2018, Alipio et al. 2019). Estos sistemas de producción se caracterizan por el control de parámetros, como la conductividad eléctrica (EC) $y$ el $\mathrm{pH}$ en las soluciones nutritivas (Palande et al.
2018), así como de otros factores, como el sustrato, agua y fertilizante (Duarte-Zaragoza et al. 2020). Además, estos sistemas permiten el abastecimiento de micronutrientes como el $\mathrm{Cu}$ (Blasco et al. 2018, Akhtar et al. 2019).

En la producción de FVH se optimizan los recursos naturales, agua, suelo y nutrientes, disminuyendo costos de producción, y optimizando los fertilizantes (Saltzman et al. 2016). Los FVH, representan mayor eficiencia en la utilización del agua, obteniendo material vegetal y rendimientos en biomasa. Al respecto, trabajos realizados en dietas con ovejas adultas, corderos en crecimiento y desarrollo, mejoraron el desempeño reproductivo y ganancias de peso (Guerrero-Cervantes et al. 2016), mientras que vacas lecheras alimentadas con $\mathrm{FVH}$ de trigo (Triticum aestivum L.) mejoraron la productividad (Harper et al. 2017). En tanto que Francis et al. (2018) mencionan que cuando las semillas germinan, la materia seca disminuye mientras que la ceniza, la fibra detergente ácida (ADF), la fibra detergente neutra (NDF), la proteína bruta (CP) y el extracto de éter (EE) aumentan, por lo que el FVH puede ser nutricionalmente superior a los forrajes secos y almacenados.

El Cu se involucra en varios procesos celulares y moleculares de las plantas, como la síntesis de clorofila, la fotosíntesis, la respiración, enzimas, metabolismo de proteínas y carbohidratos (Bashir et al. 2019). Al consumir alimentos ricos en $\mathrm{Cu}$ ayuda para el crecimiento y la reproducción normal (Etim et al. 2014, Taboada 2017). La apatita y la cal aplicadas a los suelos producen efectos sobre el $\mathrm{pH}$ disminuyendo las concentraciones de $\mathrm{Cu}$ y por el contrario con pH menores a 6 se incrementa (Neina 2019). Las prácticas agronómicas se desarrollan para ajustar el $\mathrm{pH}$ y adicionar fertilizantes quelatados como posibles soluciones (Voijant et al. 2011). En la Comarca Lagunera existe una planta que produce productos agroindustriales, entre ellos se encuentra el sulfato de cobre pentahidratado $\left(\mathrm{CuSO}_{4} .5 \mathrm{H}_{2} \mathrm{O}\right)$ $25 \%$, producto de amplio espectro de aplicaciones tanto industrial, pecuario, suplementos minerales, nutrientes vegetales, para prevenir y controlar enfermedades fungosas (Molina y Araiza 2011). Por lo 
anterior, el objetivo del presente trabajo fue evaluar la acumulación de Cu en el forraje verde hidropónico de trigo con diferentes soluciones nutritivas, fertilización con cobre y niveles de $\mathrm{pH}$.

\section{MATERIALES Y MÉTODOS}

\section{Área de estudio}

El experimento de forraje verde se realizó en febrero del 2018, en las instalaciones de la Universidad Autónoma Agraria Antonio Narro Unidad Laguna, ubicadas en la Ciudad de Torreón, Coahuila, con coordenadas geográficas $25^{\circ} 3111 \mathrm{LN}$ y $103^{\circ} 25$ 57 LO, a una altitud de 1123 msnm. El clima según Köppen modificado por García (2004) es desértico cálido, tipo BWh, con temperatura máxima de $40^{\circ} \mathrm{C}$, mínimas de $6{ }^{\circ} \mathrm{C}$, y precipitación media de $250 \mathrm{~mm}$.

\section{Obtención del forraje verde hidropónico (FVH)}

Se utilizaron 1000 semillas de trigo (Triticum aestivum L.) criolla, a las que se le aplicó una prueba con un $95 \%$ de germinación, eliminando semillas con daños, a continuación se desinfectó utilizando hipoclorito de sodio al $1 \%\left(10 \mathrm{~mL} \mathrm{~L}^{-1}\right)$, dejándose en reposo 15 minutos, finalmente se lavó con agua potable (FAO 2001) y posteriormente se colocaron en recipientes de charolas con dimensiones de $17.5 \mathrm{x}$ $12.5 \mathrm{~cm}$, a manera de siembra, elaborando una cama de $1.5 \mathrm{~cm}$ de altura, que después se envolvió en plástico negro para fomentar el crecimiento de la raíz y con la aparición de las primeras hojas, se consideró el día 0 el inicio de crecimiento de las plantas.

\section{Solución nutritiva con las modificaciones de pH}

Para obtener los tratamientos con soluciones (factor A) se utilizó el agua de la llave (ALI) Tabla 1, la solución nutritiva Steiner (Steiner 1966) (SN) + agua destilada comercial (AD) y solución nutritiva Steiner (Steiner, 1966) + agua de la llave; teniendo los tratamientos del (factor $A$ ) se procedió a obtener el (Factor B) (F) fertilizantes con $\mathrm{Cu}$, por cada tratamiento del factor A se colocaron $20 \mathrm{~mL}$ de $\mathrm{Cu}$ al $4 \%$ el cual tiene características de efecto sistémico, para el de $1.3 \%$ de Cu-EDTA (quelatado) se pesaron $1.5 \mathrm{~g}$, con respecto al Cu-EDTA de $0.28 \%$ fueron 10 g, y por último $15 \mathrm{~g}$ de Cu al $25 \%$ con características con solubilidad en agua a $20{ }^{\circ} \mathrm{C}$. Una vez agregados los fertilizantes (factor B) a las soluciones (factor A), se obtuvieron los tratamientos del $\mathrm{pH}$ (factor $\mathrm{C}$ ) modificando el pH para cada uno de los casos, para bajar el pH se aplicó hidróxido de sodio $(\mathrm{NaOH})$ hasta obtener el pH deseado, en los casos cuando el pH subía se utilizó el ácido sulfúrico $\left(\mathrm{H}_{2} \mathrm{SO}_{4}\right)$.

Tabla 1. Características físicas y químicas del agua de llave utilizada en el experimento en condiciones de invernadero.

\begin{tabular}{lc}
\hline & \\
\hline Fierro $\mathrm{Fe}^{3}$ & 0 \\
$\mathrm{Zinc} \mathrm{Zn}^{+}$ & 0.02 \\
${\text { Cobre } \mathrm{Cu}^{+}}$ & 0.03 \\
Manganeso $\mathrm{Mn}^{+}$ & 0.01 \\
Boro $\mathrm{B}^{+}$ & 0.99 \\
Sodio $\mathrm{Na}^{+}$ & 120 \\
Potasio $\mathrm{K}^{+}$ & 13 \\
${\text { Calcio } \mathrm{Ca}^{+}}^{+}$ & 288 \\
Magnesio $\mathrm{Mg}^{+}$ & 29 \\
Nitratos $\mathrm{NO}_{3}$ & 23.03 \\
Fosforo de fosfatos & 0.08 \\
Fosforo Diacino $\mathrm{H}_{2} \mathrm{PO}_{4}$ & 0.25 \\
Sulfato de $\mathrm{SO}_{4}$ & 643.6 \\
Carbonatos $\mathrm{CO}_{3}$ & 0 \\
Bicarbonatos $\mathrm{HCO}_{3}$ & 170.83 \\
Cloruros $\mathrm{Cl}$ & 198.52 \\
Parámetros físicos & \\
pH & 7.80 \\
Conductividad eléctrica mS/cm & 2.21 \\
Relación de absorción de sodio & 1.8 \\
Sodio intercambiable (\%) & 0.38 \\
\hline
\end{tabular}

\section{Riego}

Se realizaron aplicaciones de riego con aspersión manual, utilizando los tratamientos del factor $\mathrm{B}$, agregando $0.5 \mathrm{~L} \mathrm{~m}^{-1}$ durante el desarrollo del cultivo hasta la cosecha (día 14). Durante los siete días iniciales se aplicaron riegos con agua destilada (comercial), para no modificar el $\mathrm{pH}$, mediante una aplicación uniforme de la humedad, evitando la deshidratación de las semillas, al octavo día se inició con la aplicación de los tratamientos del factor $\mathrm{B}$, realizando las aplicaciones a las 08:00 am y 17:00 pm. El día de la cosecha se recolectó la biomasa verde total de cada tratamiento, se depositó en la estufa y se secaron a $65{ }^{\circ} \mathrm{C}$ durante 72 horas, para luego molerlas en un molino tipo Wiley mini-Mill de laboratorio. 


\section{Variables evaluadas}

Para medir el contenido mineral de $\mathrm{Cu}$ se realizó un análisis bioquímico a cada tratamiento, con la técnica de espectrofotometría. También se realizaron análisis bromatológicos para determinar materia seca (MS), fibra detergente neutra (FDN), fibra detergente acida (FDA), proteína cruda (PC) y cenizas (C).

\section{Análisis de cobre}

Se colectaron las muestras en verde en bolsas de papel y se colocaron dentro de la estufa a $65^{\circ} \mathrm{C}$ por 72 h. Una vez seca la muestra se procedió a molerla en un molino tipo Wiley mini-Mill para luego colocarlas en un frasco por tratamiento. Una vez separadas las muestras y tamizadas se prepararon para obtener el contenido de cobre, pesando 0.500 g para el proceso de digestión en el equipo de block digestor; posteriormente se procedió a realizar la obtención del cobre en el espectrofotómetro Milton Roy Spectronic 3000 Array, provisto de cubetas de cuarzo de $1 \mathrm{~cm}$ de paso de luz y el software Rapid Scan para el tratamiento de los espectros.

\section{Análisis bromatológico}

El contenido de materia seca (MS) y proteína cruda se determinó mediante los métodos oficiales de la AOAC (1990). Se pesó la materia seca, a los tres días después de la cosecha del FVH, se molieron los tratamientos por cada charola, y se colocaron en cápsulas de aluminio completamente secas, se pesaron $20 \mathrm{~g}$ en una balanza analítica, colocando las muestras en la estufa de aire comprimido a $65^{\circ} \mathrm{C}$ por 24 h. La PC se determinó con el equipo Micro Kjeldalh se pesaron $0.1 \mathrm{~g}$ de muestra y se colocaron en un matraz. Se cuantificó por el procedimiento Harris (1970) a partir del porcentaje del nitrógeno total y multiplicado por el factor de 5.83 (Maldonado 2011). Mientras que con el método de fraccionamiento con detergente y filtración subsecuente (Van Soest et al. 1991) se cuantificaron los contenidos de fibras. Se preparó la muestra añadiendo $100 \mathrm{~mL}$ de solución detergente ácido (SDA) al vaso de digestión. Se filtró con una tela previamente tarada en un matraz kitasato a través de un embudo de porcelana Buhner.
Una vez obtenidas las muestras se dejan secar en la estufa a $100{ }^{\circ} \mathrm{C}$ por $24 \mathrm{~h}$. Para finalizar el proceso se colocaron dentro de un desecador de vidrio por una hora y se pesaron.

Para obtener las cenizas se colocaron crisoles dentro de una mufla con muestras previamente pesadas, el procedimiento fue: los crisoles previamente secados y colocados desde un día antes en la estufa a $105{ }^{\circ} \mathrm{C}$ fueron pesados en una balanza analítica posteriormente se les agregó $2 \mathrm{~g}$ de muestra molida y secada a $65^{\circ} \mathrm{C}$, estas se metieron dentro de la mufla a $550^{\circ} \mathrm{C}$, y se dejaron durante $4 \mathrm{~h}$. Pasando las 4 $\mathrm{h}$ se sacaron y se colocaron dentro de un desecador de vidrio por una hora y se pesaron nuevamente.

\section{Análisis estadístico}

Se utilizó un arreglo factorial completamente al azar $3 \times 4 \times 3$ con tres repeticiones. Cada charola representó una unidad experimental y los factores de estudio fueron las soluciones, los fertilizantes de cobre y los niveles de $\mathrm{pH}$. Donde se consideró el factor A soluciones: (ALI, SN Steiner + AD y SN Steiner + ALI); factor $B(F)$ fertilización a base de productos de $\mathrm{Cu}(4,1.3,0.28$ y $25 \%)$ y factor $\mathrm{C}$ niveles de $\mathrm{pH}$ $(5.0,6.0$ y 7.8$)$. Los datos obtenidos fueron sometidos a análisis de varianza, realizando separación de medias mediante la prueba Tukey $(\alpha \leq 0.05)$. Para todos los procedimientos estadísiticos se utilizo el paquete estadísitico SAS (SAS 2009).

\section{RESULTADOS}

\section{Absorción de cobre en función de soluciones nu- tritivas, fertilización con cobre y $\mathbf{p H}$}

La variación en el contenido de $\mathrm{Cu}$ (ppm) en el análisis mineral, materia seca (MS\%), fibra detergente ácida (FDA\%), fibra detergente neutro (FDN\%), proteína cruda (PC\%) y ceniza (\%) fue significativa $(p \leq 0.05)$. El análisis de varianza individual para ppm de $\mathrm{Cu}, \mathrm{MS}$, FDA, FDN, PC y cenizas detecto diferencias altamente significativa para los efectos principales; solución, $p=0.0002-0.001$, excepto en MS y proteína cruda $p=0.3581-0.4856$; porcentaje de fertilizantes de cobre, $p=0.001$; excepto MS $p=$ $0.1884 ; \mathrm{pH}, \mathrm{p}=0.001-0.0017$, excepto MS y cenizas 
$p=0.1039-0.1305$. En las combinaciones de $S x$ $\mathrm{F}, \mathrm{p}=0.0002-0.001 ; \mathrm{S} \times \mathrm{pH}, \mathrm{p}=0.001-0.0047$, excepto MS, $\mathrm{p}=0.3201 ; \mathrm{F} \times \mathrm{pH}, \mathrm{p}=0.0001-0.001 ; \mathrm{S}$ $x \mathrm{~F} \times \mathrm{pH}, \mathrm{p}=0.001$ excepto MS, $\mathrm{p}=0.0846$, los coeficiente de variación oscilaron entre $2.0-7.76 \%$. En las Figuras 1 y 2 se observa, la absorción de cobre en $\mathrm{FVH}$, con respecto a las interacciones de soluciones $\mathrm{x}$ fertilización con $\mathrm{Cu}$ y soluciones por $\mathrm{pH}$. La mayor absorción de Cu se tuvo con las combinaciones de las soluciones nutritivas y la fertilización con 1.3\% de $\mathrm{Cu}$, con concentración media de 5.04 ppm de $\mathrm{Cu}$ en el FVH para las distintas soluciones nutritivas y una concentración máxima de 5.51 ppm con agua de llave (ALI). En la Figura 2 se observa que las combinaciones de soluciones nutritivas y $\mathrm{pH}$ de 6.0 presentaron mayores absorciones por el $\mathrm{FVH}$, con una media de 3.49 ppm y concentración máxima de 3.49 ppm con la solución AD + SN.

\section{Relaciones entre el contenido de cobre y las variables de calidad de forraje}

El efecto del contenido de $\mathrm{Cu}$ en las variables de calidad del forraje FDA, PC y FDN para fertilización con $\mathrm{Cu}$, SN y la combinación de fertilización con $\mathrm{Cu}$ y $\mathrm{pH}$ se muestra en las Figura 3 donde se observa una estrecha relación entre la absorción de Cu y el \% de FDA, presentando una tendencia lineal positiva y significativa $(r=0.9735, p=0.0334)$ para los diferentes niveles de fertilización con Cu (Figura 3a). En los tres niveles de solución, se presentó una tendencia lineal negativa y significativa $(r=0.9934, p=0.0238)$ entre el contenido de $\mathrm{Cu}$ y el \% de PC (Figura $3 b$ ). En la combinación de fertilización con $\mathrm{Cu}$ y $\mathrm{pH}$, se tuvo una tendencia lineal positiva y significativa $(r=0.634, p$ $=0.023$ ) en la relación entre contenido de Cu y el \% de FDN (Figura 3c). En la Figura 3d se muestra la relación entre el \% de FDN y \% de PC para la combinación de solución y $\mathrm{pH}$, la cual fue positiva y significativa $(r=0.672, p=0.0072)$. En las Figuras 3 e y $3 f$ se muestran las relaciones entre el contenido de PC y las cenizas para las combinaciones de soluciones y fertilización con $\mathrm{Cu}$, así como para la combinación de fertilización con $\mathrm{Cu}$ y $\mathrm{pH}$, respectivamente, en ambas se presentaron tendencias negativas y significativas $(r=0.713$ y $0.7293, p=0.0092$ y 0.0072 , respectivamente). Es interesante considerar que las variables $\%$ de FDA y $\%$ de PC del forraje fueron afectadas positiva y negativamente por la absorción de $\mathrm{Cu}$, respectivamente; por lo que podría ser útil considerar estos resultados en estudios futuros sobre los caracteres químicos relacionados con la digestibilidad en rumiantes. Uno de los aspectos importantes sería investigar más sobre la biofortificación, el diseño y generación de técnicas que podrían ayudar en el balanceo de las dietas y la nutrición animal.

\section{DISCUSIÓN}

\section{Absorción de cobre}

Se encontró mayor absorción de Cu en el FVH con agua de la llave, fertilización con Cu-EDTA al $1.3 \%$ y $\mathrm{pH}$ de 6 ; estudios realizados por Alva et al. (2000) y Acosta et al (2019) en trigo (Triticum aestivum L.) con pH de 4.6 a 6.4 reportan resultados similares, mientras que Palande et al. (2018), Alipio et al. (2019) y Cui et al. (2019) señalan que pH menores favorecen la absorción de $\mathrm{Cu}$, en tanto que incrementos del pH de 4.6 a 7 aumenta la adsorción de $\mathrm{Cu}$, y que con un $\mathrm{pH}$ inferior a 5.5 se afecta el comportamiento de los metales traza. Por otro lado DíazGómez et al. (2017) y Akhtar et al. (2019) señalan que la acumulación de nutrientes en la planta pueden transportarse fácilmente al endospermo mediante la aplicación foliar. Otros estudios en Albahaca, señala que el sulfato de cobre $\left(\mathrm{CuSO}_{4}\right)$ tiene efectos benéficos en sus componentes de rendimiento (Rezende et al. 2018); mientras que Chairi et al. (2010) y Acosta et al. (2019), hacen referencia que elevan sus niveles de absorción, bioacumulación y fertilización. La mayor absorción de Cu y disponibilidad de micronutrientes cuando el $\mathrm{pH}$ fue modificado para disolver tanto el $\mathrm{Cu}$ y minerales, concuerda con datos de Dimkpa y Bindraban (2016) y Blasco et al. (2018) quienes mencionan que la absorción de $\mathrm{Cu}$ tiene relación con mecanismos moleculares a base de quelación. 

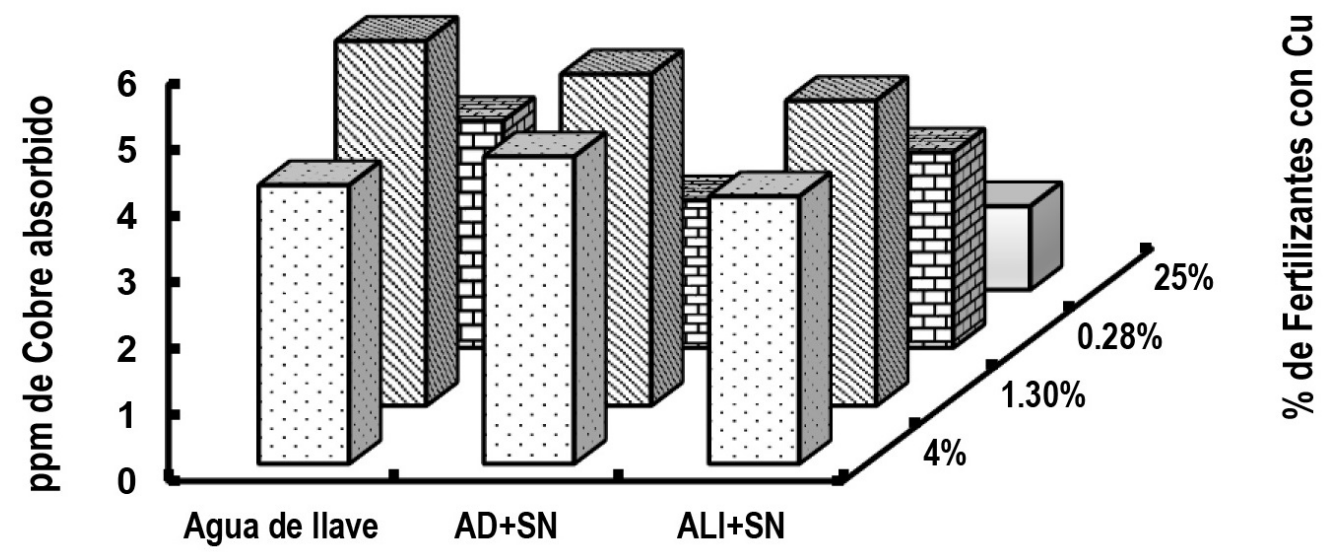

Soluciones

Figura 1. Absorción de cobre por el forraje en función de soluciones nutritivas y fertilización con cobre.

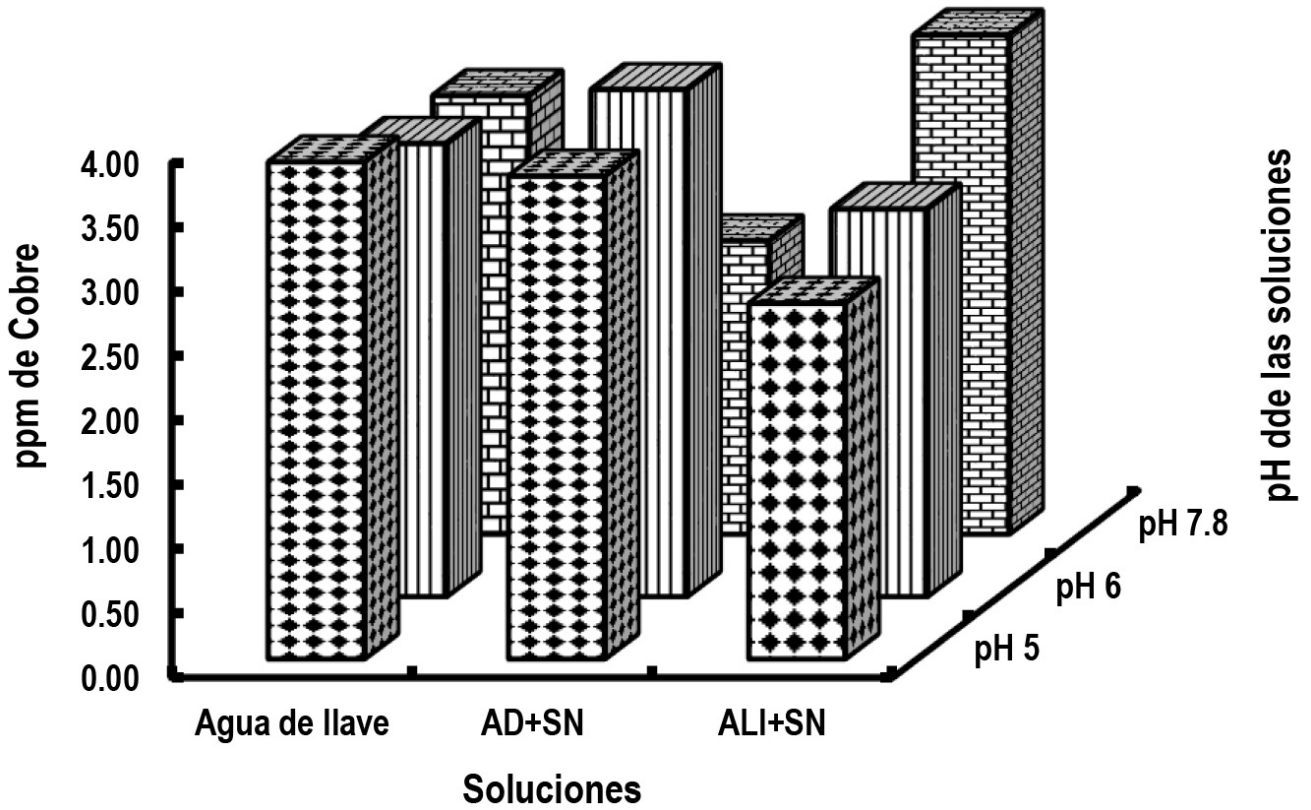

Figura 2. Absorción de cobre por el forraje en función de las soluciones nutritivas y pH.

\section{Análisis bioquímico bromatológico}

De acuerdo a Herrera et al. (2010), valores de FDN mayores al $60 \%$ son altos y pudieran interferir con el consumo y la digestión, en este estudio correspondieron de un 81 a $56 \%$ cuando se aplicó fertilizante a las diferentes soluciones, mientras que para FDA debe ser entre un 19 y $27 \%$ NRC (2001) en este caso correspondieron de un 13 a $24 \%$ cuando se aplicó solución con fertilizante y se modificó el pH. Broadley et al. (2012) señalan que el contenido de FDA aumenta con el desarrollo de la planta por el efecto de la maduración y al aplicar el Cu este interfirió en el proceso de lignificación, que es la fracción insoluble de la planta. Francis et al. (2018) señalan que el manganeso (Mn) y el $\mathrm{Cu}$ ayudan en los procesos de los sistemas redox, como activadores de 

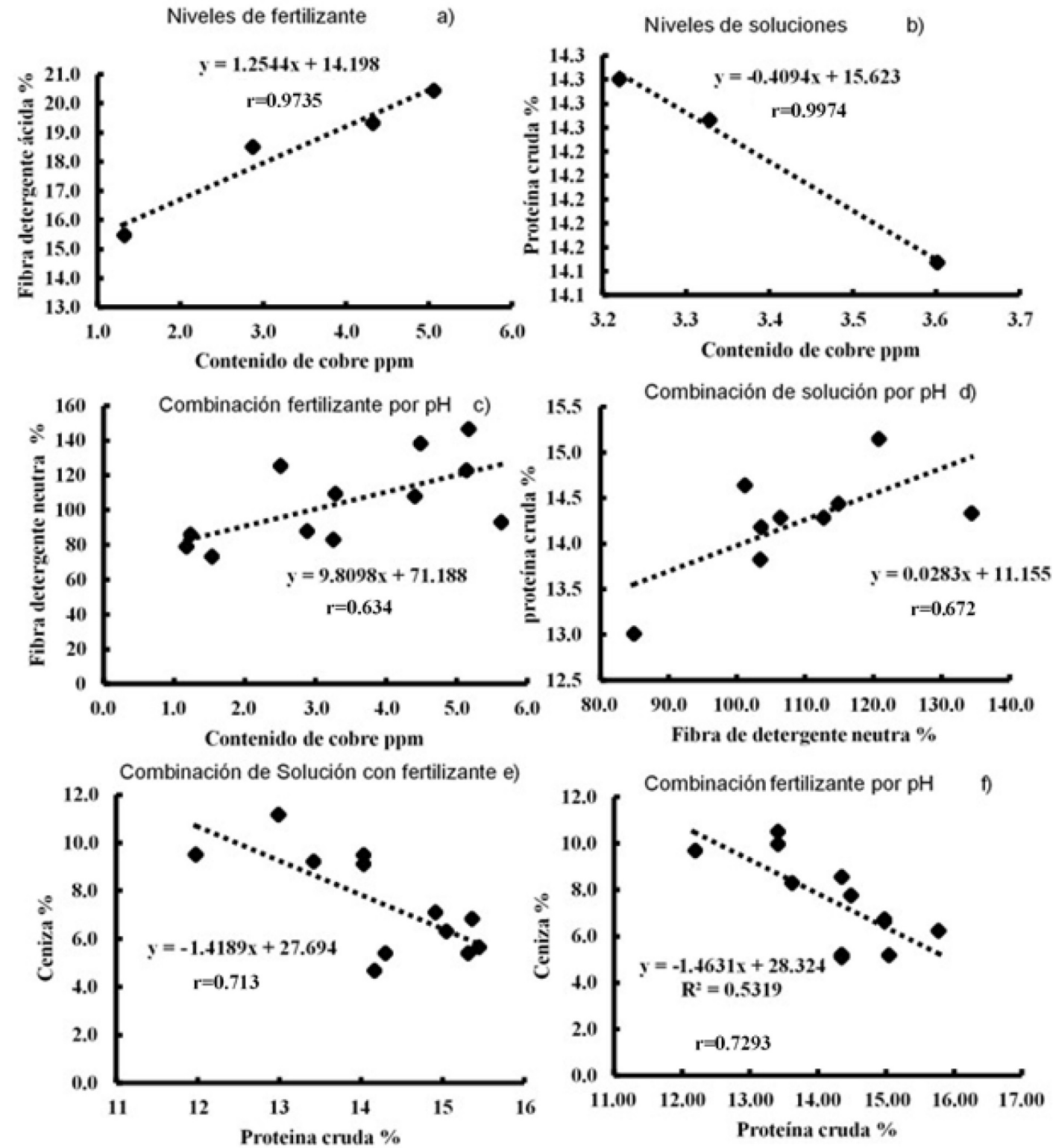

Figura 3. Relaciones entre contenido de cobre en el forraje y \% de FDA para diferentes niveles de fertilización con cobre (a), $\%$ de PC para las diferentes soluciones (b) y \% de FDN para la combinación de fertilización con cobre y pH (c), entre \% de FDN y \% de PC para la combinación de soluciones y pH (d), entre \% de PC y \% de ceniza para la combinación de soluciones y fertilización con cobre (e) y entre \% de PC y \% de ceniza para la combinación de fertilización con cobre y pH (f).

enzimas que están involucradas con la lignina.

\section{Relación entre el contenido de Cu y las variables de calidad del forraje}

En la combinación de soluciones con diferentes $\mathrm{pH}$, se observó que el porcentaje de PC presentó una relación positiva y significativa con el porcentaje de FDN; mientras que en la combinación de soluciones y fertilizante el porcentaje ceniza se relacionó de forma negativa y significativa con el porcentaje de PC (Figura 3). Al incrementarse la absorción de $\mathrm{Cu}$ se incrementa el porcentaje FDN mientras que FDA se mantiene estable, estos resultados son similares los reportados por Watson et al. (2012), 
Harper et al. (2017), Acosta et al. (2019), Blasco et al. (2018), y Akhtar et al. (2019) aunque reduce el porcentaje de PC, demostrando que el $\mathrm{pH}$ es un factor importante para determinar la absorción de $\mathrm{Cu}$ en el FVH así como el Cu en las variables de calidad del forraje.

\section{CONCLUSIONES}

La absorción de Cu por el FVH de trigo fue afectada por la solución nutritiva, fertilización con $\mathrm{Cu}$ y $\mathrm{pH}$. Las mayores concentraciones de cobre se tuvieron con la fertilización de Cu-EDTA al 1.3\%, con máxima concentración de $5.51 \mathrm{ppm}$. El pH de 6 presentó las mayores concentraciones de $\mathrm{Cu}$, con la solución de agua destilada. La combinación de agua de la llave con los fertilizantes a base de Cu-
EDTA al $1.3 \%$ y pH de 6 , es lo más recomendables para incrementar la absorción de cobre en el FVH. El contenido de cobre en el forraje incremento el contenido de FDN mientras que FDA se mantiene dentro de los parametros. Estos resultados pueden ser de utilidad en estudios futuros para realizar más ensayos sobre los caracteres bioquímicos relacionados con la digestibilidad de forrajes y la biofortificación agronómica.

\section{AGRADECIMIENTOS}

Al laboratorio y departamento de Suelos, laboratorio de Bromatología del departamento de Producción Animal de la Universidad Autónoma Agraria Antonio Narro.

\section{LITERATURA CITADA}

Acosta-Estrada BA, Gutiérrez-Uribe JA, Serna-Saldivar SO (2019) Minor constituents and phytochemicals of the kernel. In: Serna-Saldivar SO (ed). Corn: Chemistry and technology. Third Edition. AACC International. Elsevier. UK. pp: 369-403.

Akhtar S, Ismail T, Hussain M (2019) Micronutrient fortification of flours developing countries' perspective. In: Preedy VR, Watson RR (ed). Flour and breads and their fortification in health and disease prevention. Academic Press. Pakistán. pp: 263-271.

Alipio MI, De la Cruz AEM, Doria JDA, Fruto RMS (2019) On the design of nutrient film technique hydroponics farm for smart agriculture. Engineering in Agriculture, Environment and Food 12: 315-324.

Alva AK, Huang B, Paramasivam S (2000) Soil pH affects copper fractionation and phytotoxicity. Soil Science Society of America Journal 64: 955-962.

AOAC (1975) Official Methods of Analysis of the Association of Official Agricultural Chemists. AOAC International. Washington, D.C. USA. 70p.

Bashir K, Seki M, Nishizawa NK (2019) The transport of essential micronutrients in rice. Molecular Breeding 168: 1-17.

Blasco B, Navarro-León E, Ruiz JM (2018) Oxidative stress in relation with micronutrient deficiency or toxicity. In: Hossain MA, Kamiya T, Tran LP, Fijiwara T (ed) Plant micronutrient use ffficiency. Academic Press. Spain. pp: 181-194.

Broadley M, Brown P, Cakmak I, Rangel Z, Zhao F (2012) Function of nutrients: micronutrients. In: Marschner P (ed). Mineral nutrition of higher plant. AACC International. Elsevier. UK. pp: 191-248.

Chairi H, Fernández-Díaz C, Navas JI, Machado M, Rebordinos L, Blasco J (2010) In vivo genotoxicity and stress defences in three flatfish species exposed to $\mathrm{CuSO}_{4}$. Ecotoxicology and Environmental Safety 73: $1279-1285$. 
Chugh V, Dhaliwal HS (2013) Biofortification of staple crops. In: Bhullar SG, Bhullar NK (ed). Agricultural sustainability. AACC International. Elsevier. UK. pp: 177-196.

Cui j, Zhao Y, Lu Y, Chan T, Zhang L, Tsang DCW, Li XD (2019) Distribution and speciation of copper in rice (Oryza sativa L.) from miningimpacted paddy soil: Implications for copper uptake mechanisms. Enviroment International 126: 717-726.

Díaz-Gómez J, Twyman RM, Zhu Ch, Farré G, Serrano JCE, Portero-Otin M, Muñoz P, Sandmann G, Capell T, Christou P (2017) Biofortification of crops with nutrients: factors affecting utilization and storage. Current Opinion in Biotechnology 44: 115-123.

Dimkpa CO, Bindraban PS (2016) Fortification of micronutrients for efficient agronomic production: a review. Agronomy for Sustainable Development 36: 7. DOI: 10.1007/s13593-015-0346-6.

Duarte-Zaragoza VM, Pérez-Hernández VS, Hernández-Acosta E, Villanueva-Morales A (2020) Exploratory study of lead and copper accumulation in Prosopis laevigata in mine tailings. Ecosistemas y Recursos Agropecuarios 7: e2311. DOI: 10.19136/era.a7n2.2311.

Etim N, Enyenihi GE, Akpabio U, Offiong EEA (2014) Effects of nutrition on haematology of rabbits: A Review. European Scientific Journal 10: 413-424.

FAO (2001) Manual Técnico Forraje Verde Hidropónico. FAO. Santiago, Chile. 55p.

Francis JM, Apgar GA, Crandell KG, Handlos GC, Perry EB (2018) The effects of hydroponic wheat fodder on fecal metabolites in equines. Journal of Equine Veterinary Science 70: 84-90.

García AE (2004) Modificaciones al Sistema de Clasificación Climática de Köppen para Adaptarlo a las Condiciones de la República Mexicana. Offset Larios. México. 246p.

García CM, Luna OJG, González TA, González ZA, Gallegos RMG, Vázquez VC, Cervantes VMG, González SU (2017) Relationship of dieldrin and soil properties in Comarca Lagunera, México. Revista Mexicana de Ciencias Agrícolas 8: 1691-1703.

García-González CY, Moreno-Reséndez A, Figueroa-Viramontes U, Reyes-Carrillo JL (2020) Accumulation of heavy metals in irrigated alfalfa with wastewater from the textile industry. Ecosistemas y Recursos Agropecuarios 7: e2437. DOI: 10.19136/era.a7n2.2437.

Garg M, Sharma N, Sharma S, Kapoor P, Kumar A, Chunduri V, Arora P (2018) Biofortified crops generated by breeding, agronomy, and transgenic approaches are improving lives of millions of people around the word. Frontiers in Nutrition 5: 12. DOI: 10.3389/fnut.2018.00012.

Guerrero-Cervantes M, Cerrillo-Soto MA, Plascencia A, Salem AZM, Estrada-Angulo A, Rios-Rincón FG, Luginbuhl JM, Bernal-Barragán H, Abdalla AL (2016) Productive and reproductive performance and metabolic profiles of ewes supplemented with hydroponically grown green wheat (Triticum aestivum L.). Animal Feed Science and Technology 221: 206-214.

Harper MT, Oh J, Giallongo F, Roth GW, Hristov N (2017) Inclusion of wheat and triticale silage in the diet of lactating dairy cows. Journal of Dairy Science 100: 6151-6163.

Harris LE (1970) Métodos para el análisis químico y la evaluación biológica de alimentos para animales. Gainesville: Center for Tropical Agriculture, University of Florida. Florida. USA. 174p.

Herrera-Torres E, Cerrillo-Soto MA, Juárez-Reyes MA, Murillo-Ortiz AS, Ríos-Rincón M, Reyes-Estrada FG, Bernal-Barragán H (2010) Efecto del tiempo de cosecha sobre el valor proteico y energético del forraje verde hidropónico de trigo. Interciencia 35: 284-289. 
Maldonado TR, Álvarez SME, Acevedo DC, Ríos SE (2013) Nutrición mineral de forraje verde hidropónico. Revista Chapingo Serie Horticultura 19: 211-223.

Masunaga T, Marques FJD (2018) Strategies for increasing micronutrient availability in soil for plant uptake. In: Mohammad AH, Takehiro K, Burrit DJ, Phan TLS, Fijiwara T (ed). Plant micronutrient use efficiency. Academic Press. Japan. pp: 195-208.

Medina MMC, Medina MEJ, Aguilar PJH, García GSJ (2000) Manganese and copper foliar sprays in pecan trees. Terra Latinoamericana 17: 317-323.

Molina JC, Araiza SE (2011) Peñoles en la agroindustria empresa orgullosamente mexicana. Revista Mexicana de Agronegocios 28: 490-501.

Moreno PEC, Sánchez CF, Gutiérrez TJ, González ML, Pineda PJ (2015) Greenhouse lettuce production with and without nutrient solution recycling. Revista Chapingo Serie Horticultura 21: 43-55.

Neina D (2019) The role of soil pH in plant nutrition and soil remediation. Applied and Environmental Soil Science 2019: ID: D 5794869. DOI: 10.1155/2019/5794869.

NRC (2001) Nutrient requirements of dairy cattle. Seventh Edition. National Academy Press. Washington, DC USA 251p.

Palande V, Zaheer A, George K (2018) Fully automated hydroponic system for indoor plant growth. Procedia Computer Science 129: 482-488.

Rezende TJ, Cristiani GZ, Goncalvez JE, Straicieri J, Magalhãesa HM (2018) Effects of copper sulphate $\left(\mathrm{CuSO}_{4}\right)$ elicitation on the chemical constitution of volatile compounds and the in vitro development of Basil. Scientia Horticulture 234: 19-26.

Saltzman A, Anderson MS, Asare-Marfo D, Lividini K, De Moura FF, Moursi M, Oparinde A, Taleon V (2016) Biofortification techniques to improve food security. Reference Module in Food Science. Elsevier. DOI: 10.1016/b978-0-08-100596- 5.03078-x. Fecha de consulta: 6 de septiembre del 2021.

SAS (2009) SAS Institute. SAS/STATR (99.2. User Guide Release. Cary, NC: SAS Institute Inc. USA. 111p.

Steiner AA (1966) The influence of the chemical composition of a nutrient solution on the production of tomato plants. Plant and Soil 3: 454-466.

Taboada LN (2017) El zinc y el cobre: micronutrientes esenciales para la salud humana. Acta Médica del Centro 11: 79-89.

Van Soest PJ, Robertson JB, Lewis BA (1991) Methods for Dietary Fiber, Neutral Detergent Fiber, and Nonstarch Polysaccharides in Relation to Animal Nutrition. Journal of Dairy Science 74: 35-83.

Voijant TB, Sheikh ASR, Basri H, Idris M, Anuar N, Mukhlisin M (2011) A review on heavy metals (As, Pb, and $\mathrm{Hg}$ ) uptake by plants through phytoremediation. International Journal of Chemical Engineering 2011: 1-31.

Watson CA, Öborn I, Edwards AC, Dahlin AS, Eriksson J, Lindström BEM, Linse L, Owens K, Topp CFE, Walker $\mathrm{RL}$ (2012) Using soil and plant properties and farm management practices to improve the micronutrient composition of food and feed. Journal of Geochemical Exploration 121: 15-24.

Yescas CP, Álvarez RVP, Segura CMA, García CM, Hernández HV, González CG (2018) Variabilidad espacial del carbono orgánico e inorgánico del suelo en la Comarca Lagunera, México. Boletín de la Sociedad Geológica Mexicana 70: 591-610. 